\title{
THE WEAK CARBON MONOXIDE EMISSION IN AN EXTREMELY METAL-POOR GALAXY, SEXTANS A*
}

\author{
Yong Shi ${ }^{1,2,3}$, JunZhi Wang ${ }^{4,5}$, Zhi-Yu Zhang ${ }^{6,7}$, Yu GaO ${ }^{8}$, Lee Armus ${ }^{9}$, \\ George Helou ${ }^{9}$, Qiusheng Gu ${ }^{1,2,3}$, and Sabrina Stierwalt ${ }^{10}$ \\ ${ }^{1}$ School of Astronomy and Space Science, Nanjing University, Nanjing 210093, China; yshipku@ gmail.com \\ ${ }^{2}$ Key Laboratory of Modern Astronomy and Astrophysics, Nanjing University, Ministry of Education, Nanjing 210093, China \\ ${ }^{3}$ Collaborative Innovation Center of Modern Astronomy and Space Exploration, Nanjing 210093, China \\ ${ }^{4}$ Shanghai Astronomical Observatory, Chinese Academy of Sciences, 80 Nandan Road, Shanghai 200030, China \\ ${ }^{5}$ Key Laboratory of Radio Astronomy, Chinese Academy of Sciences, Nanjing 210008, China \\ ${ }^{6}$ Institute for Astronomy, University of Edinburgh, Royal Observatory, Blackford Hill, Edinburgh EH9 3HJ, UK \\ ${ }^{7}$ ESO, Karl Schwarzschild Strasse 2, D-85748 Garching, Munich, Germany \\ ${ }^{8}$ Purple Mountain Observatory, Chinese Academy of Sciences, 2 West Beijing Road, Nanjing 210008, China \\ ${ }^{9}$ Infrared Processing and Analysis Center, California Institute of Technology, 1200 E. California Boulevard, Pasadena, CA 91125, USA \\ ${ }^{10}$ Department of Astronomy, University of Virginia, P.O. Box 400325, Charlottesville, VA 22904, USA \\ Received 2015 January 21; accepted 2015 April 3; published 2015 April 24
}

\begin{abstract}
Carbon monoxide $(\mathrm{CO})$ is one of the primary coolants of gas and an easily accessible tracer of molecular gas in spiral galaxies, but it is unclear if CO plays a similar role in metal-poor dwarfs. We carried out a deep observation with IRAM $30 \mathrm{~m}$ to search for CO emission by targeting the brightest far-IR peak in a nearby extremely metal-poor galaxy, Sextans A, with 7\% solar metallicity. A marginal signal of CO $J=1-0$ emission is seen, which is already faint enough to place a strong constraint on the conversion factor $\left(\alpha_{\mathrm{CO}}\right)$ from the $\mathrm{CO}$ luminosity to the molecular gas mass that is derived from the spatially resolved dust-mass map. The $\alpha_{\mathrm{CO}}$ is at least seven hundred times the Milky Way value. This indicates that CO emission is exceedingly weak in Sextans A, challenging its role as a coolant in extremely metal-poor galaxies.
\end{abstract}

Key words: galaxies: dwarf - galaxies: ISM - submillimeter: ISM

\section{INTRODUCTION}

Stars form out of molecular clouds (Kennicutt 1998a; Gao \& Solomon 2004). The efficient cooling of molecular gas is the prerequisite for gas collapse and star formation. Among molecular species, carbon monoxide (CO) plays an important role in cooling molecular gas in the low temperature and density regime (Goldsmith 2001). The CO emission is thus related to the fundamental question about how stars form out of gas. $\mathrm{CO}$ cools gas by radiation, and its bright emission renders $\mathrm{CO}$ the most common tracer of the molecular gas mass (Young \& Scoville 1982), while the two most abundant components, $\mathrm{H}_{2}$ and $\mathrm{He}$, cannot be observed directly in emission at the characteristic temperature of molecular gas clouds. Over the past decade, the $\mathrm{CO}$ emission has been detected in galaxies with increasingly lower metallicity (Israel 1997; Taylor et al. 1998; Leroy et al. 2007, 2011), down to $15 \%$ solar (Elmegreen et al. 2013). The conversion factor from $\mathrm{CO}$ to $\mathrm{H}_{2}$ (referred as $\alpha_{\mathrm{CO}}$ ) has been constrained by comparing to the molecular gas mass inferred from other methods, showing values 10-100 times larger than the Milky Way value (Bolatto et al. 2013) in low-metallicity environments. Probing CO emission at lower metallicities establishes if $\mathrm{CO}$ emission can be an efficient gas coolant and effective tracer of molecular gas in metal-poor galaxies both locally and in the early universe.

Sextans $\mathrm{A}$ is an irregular dwarf at $1.4 \mathrm{Mpc}$ with an oxygen abundance of $7 \%$ solar (Pettini \& Pagel 2004; Kniazev et al. 2005). Its proximity increases the detectability of potential CO emission in the galaxy. It is one of a few extremely metal-poor galaxies whose molecular gas masses have been estimated through the spatially resolved dust map

\footnotetext{
* Based on observations carried out with the IRAM $30 \mathrm{~m}$ Telescope. IRAM is supported by INSU/CNRS (France), MPG (Germany), and IGN (Spain).
}

(Shi et al. 2014), arguably the most accessible way to measure cold gas mass in metal-poor galaxies (Bolatto et al. 2013). The data are presented in Section 2. The results and discussion are given in Sections 3 and 4, respectively. Conclusions are in Section 5.

\section{OBSERVATIONS}

The CO $J=1-0$ observations toward the star formation region (R.A.: 10:11:06.55, decl.: -04:42:04.70, J2000) in Sextans A were done from 2014 August 23 to 25, using the IRAM $30 \mathrm{~m}$ millimeter telescope at Pico Veleta, Spain. The target region, which is $22 \operatorname{arcsec}$ as the IRAM beam size at this frequency, is the far-IR peak as shown in Figure 1. The Eight Mixer Receiver with dual-polarization and the Fourier Transform Spectrometers backend were used, which gave the frequency channel spacing of $195 \mathrm{KHz}$. The standard wobbler switching mode with a $\pm 120^{\prime \prime}$ offset at $0.5 \mathrm{~Hz}$ beam throwing was used for the observations. Pointing and focusing were checked about every $2 \mathrm{hr}$ with nearby strong millimeter emitting quasi-stellar objects. We read out the spectra every 2 minutes, while the typical system temperature $\left(T_{\text {sys }}\right)$ was about $280 \mathrm{~K}$ at this band. Data reduction was conducted with the CLASS in GILDAS software package. We checked each spectrum, and only used the spectra with $T_{\text {sys }}$ less than $280 \mathrm{~K}$ for the final discussion. After throwing out about $50 \%$ of the spectra, the total source time was about $5 \mathrm{hr}$, which gave the noise level of about $4.1 \mathrm{mK}$ in the main beam temperature after smoothing the frequency resolution to $6.2 \mathrm{MHz}\left(\sim 16 \mathrm{~km} \mathrm{~s}^{-1}\right)$.

The Herschel data at 70,160, 250, and $350 \mu \mathrm{m}$ and the Spitzer data at 3.6, 4.5, 5.6, 8.0, and $24 \mu \mathrm{m}$ are from Shi et al. (2014) and Dale et al. (2009), respectively. Because the dust emission within the IRAM beam is partially resolved instead of a simple point-spread function, to measure the photometry 


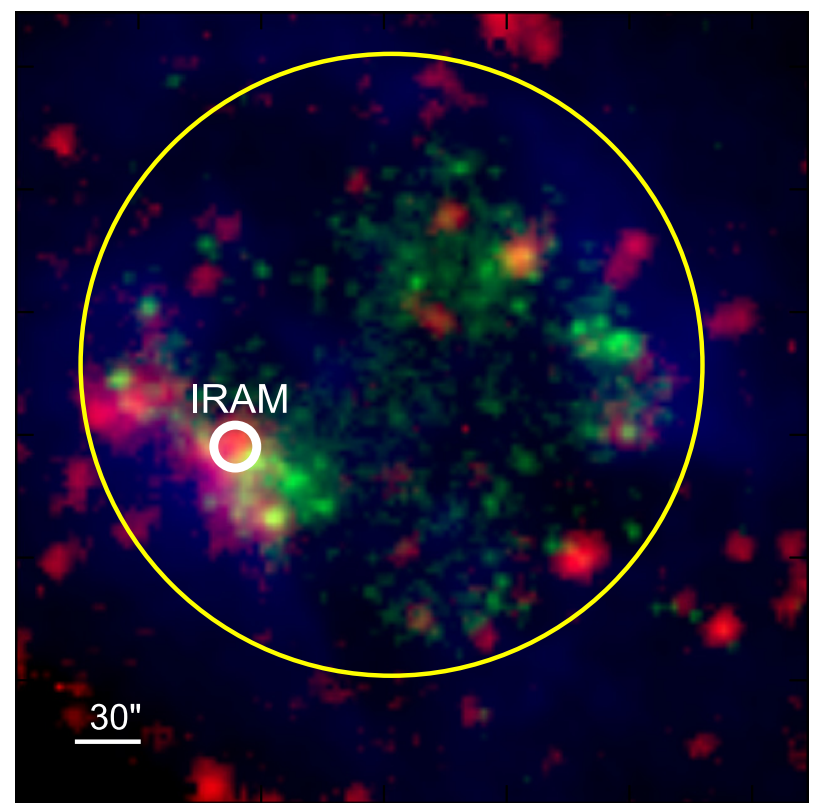

Figure 1. False-color, multi-wavelength images of Sextans A. Red is the sum of Herschel 160 and $250 \mu \mathrm{m}$ data, green is GALEX far-UV, and blue is radio $21 \mathrm{~cm}$ data showing the $\mathrm{HI}$ atomic gas. The IRAM $30 \mathrm{~m}$ pointing at $\mathrm{CO}$ $J=1-0$ with the beam size is indicated by the white circle, while the yellow circle indicates the star-forming disk defined at a surface brightness of $25.9 \mathrm{ABmag} \operatorname{arcsec}^{-2}$ in the GALEX far-UV band.

within the IRAM beam, we first convolved all images to the $350 \mu \mathrm{m}$ resolution based on the convolution Kernels (Aniano et al. 2011). Aperture photometry at all bands was then measured at this spatial resolution. The corresponding aperture correction factor is taken as the ratio of the $70 \mu \mathrm{m}$ photometry at its native resolution to that at the $350 \mu \mathrm{m}$ resolution by assuming that the $70 \mu \mathrm{m}$ map at its native resolution can resolve the dust structure. We further included the farultraviolet (UV) images from the GALEX Space Telescope archive as well as maps of atomic gas (Ott et al. 2012).

\section{RESULTS}

Figure 2 shows the observed $\mathrm{CO} J=1-0$ spectrum at a resolution of $16 \mathrm{~km} \mathrm{~s}^{-1}$ with $c z=324 \mathrm{~km} \mathrm{~s}^{-1}$. A marginal signal at $350 \mathrm{~km} \mathrm{~s}^{-1}$ was seen. A Gaussian fitting gives a signal-to-noise ratio of 3.7 for the integrated flux, a width of $21 \pm 12 \mathrm{~km} \mathrm{~s}^{-1}$, and a peak intensity of $6.3 \mathrm{mK}$ while the $1 \sigma$ noise is $1.4 \mathrm{mK}$. The distribution of the noise level is slightly non-Gaussian. Using the negative values of channels over a velocity range from -500 to $1500 \mathrm{~km} \mathrm{~s}^{-1}$, we estimated a probability of $0.05 \%$ for the signal to be artificial, which corresponds to a $3.4 \sigma$ significance for a Gaussian distribution of noises. The feature lies within the velocity range of atomic gas as shown in Figure 2. The offset in the velocity between the two lines may be reasonable as the two trace different phases of the ISM. As listed in Table 1, the intensity $I_{\mathrm{CO}(J=1-0)}$ is $145 \pm 39 \mathrm{mK} \mathrm{km} \mathrm{s}^{-1}$ in $T_{\mathrm{mb}}$, corresponding to a point-source luminosity (Gao \& Solomon 2004) of $\sim(3.7 \pm 1.0) \times 10^{3} \mathrm{~K} \mathrm{~km} \mathrm{~s}^{-1} \mathrm{pc}^{2}$ for a beam size of 22 arcsec. This is much smaller than the previous upper limits (Taylor et al. 1998) of a few $\times 10^{4} \mathrm{~K} \mathrm{~km} \mathrm{~s}^{-1} \mathrm{pc}^{2}$ observed at the position (R.A. $=10^{\mathrm{h}} 11^{\mathrm{m}} 07^{\mathrm{s}} .3$, decl. $=-4^{\mathrm{d}} 42^{\mathrm{m}} 22^{\mathrm{s}} .5$, J2000) near our pointing with a beam size of 55 arcsec. The $3 \sigma$ CO $J=2-1$ assuming the same velocity dispersion as $\mathrm{CO} J=1-0$ is $460 \mathrm{mK} \mathrm{km} \mathrm{s}^{-1}$.

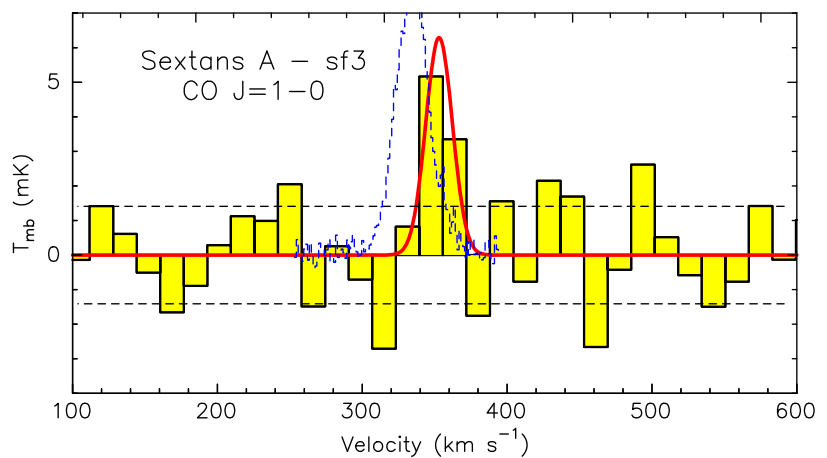

Figure 2. Submillimeter spectrum around the $\mathrm{CO} J=1-0$ frequency with the source redshift $c z=324 \mathrm{~km} \mathrm{~s}^{-1}$. The red Gaussian profile is the fit to the signal at $350 \mathrm{~km} \mathrm{~s}^{-1}$, which gives a signal-to-noise ratio of 3.7 for the integrated flux. The two black dotted lines are the $1 \sigma$ noise levels. The blue curve indicates the velocity of the HI emission line within the IRAM beam as measured from the HI data but with the absolute intensity scaled arbitrarily (Ott et al. 2012).

The conversion factor $\alpha_{\mathrm{CO}}$ from $\mathrm{CO}$ to $\mathrm{H}_{2}$ is quantified by comparing the $\mathrm{CO}$ luminosity to the molecular gas mass that is inferred from the spatially resolved dust map (Israel 1997; Leroy et al. 2011; Sandstrom et al. 2013). As shown in Figure 3, the infrared spectral energy distribution (SED) is first fitted with the model of Draine \& Li (2007), as detailed in Shi et al. (2014), based on that which the total cold gas $\left(\mathrm{HI}+\mathrm{H}_{2}\right)$ within the IRAM beam is measured by multiplying the dust mass with the gas-to-dust ratio derived from the diffuse regions of the galaxy. As discussed in Shi et al. (2014), the derived total cold gas mass is not sensitive to the absolute dust mass but to the ratio of dust masses of star-forming and diffuse regions. As a result, as long as diffuse and star-forming regions have similar dust grains, different dust models gives a similar estimate of cold gas masses within the IRAM beam. To derive the gas mass of star-forming regions, we do assume starforming regions have the same gas-to-dust ratio as the diffuse regions, which is true for spiral galaxies (e.g., Sandstrom et al. 2013). However, this assumption may break down in dwarfs as their star formation history is stochastic in both space and time so that both diffuse and star-forming regions are affected by different physical processes that impact the gas-todust ratio. For example, the $\mathrm{SN}$ shocks may continuously destroy the dust in the diffuse regions in the past, meanwhile dust grains in dense clouds are protected from such destruction but instead grow further. Nevertheless, such a suspect needs systematic investigations before any conclusions can be made.

After subtracting the atomic gas mass from the derived total gas mass, the molecular gas mass is shown to be about $10^{7} M_{\odot}$ as listed in Table 1 . The derived $\alpha_{\mathrm{CO}}$ value is about $(2.8 \pm 1.1) \times 10^{3} M_{\odot} \mathrm{pc}^{-2}\left(\mathrm{~K} \mathrm{~km} \mathrm{~s}^{-1}\right)^{-1}, \sim 700$ times larger than the Milky Way value $\left(4.3 M_{\odot} \mathrm{pc}^{-2}\left(\mathrm{~K} \mathrm{~km} \mathrm{~s}^{-1}\right)^{-1}\right.$ (Bolatto et al. 2013). In Figure 4, we compared our measurement to those in the literature (Israel 1997; Leroy et al. 2011; Sandstrom et al. 2013), whose molecular gas masses are derived in the same way as ours except for the galaxy WolfLundmark-Melotte (WLM; Elmegreen et al. 2013). The gasto-dust ratio used to derive the $\alpha_{\mathrm{CO}}$ of WLM is based on the relationship between $\alpha_{\mathrm{CO}}$ and metallicity (Rémy-Ruyer et al. 2014), which shows a large scatter $(>10)$ around the metallicity of WLM. The metallicity measurement of Sextans A was not taken exactly at the $\mathrm{CO}$ pointing. However, as no metallicity gradient is seen in Sextans A (Kniazev et al. 2005), we did not expect large offsets $(>0.1 \mathrm{dex})$ from the value 
Table 1

The Properties of the Dwarf Galaxy Sextans A with the IRAM CO (1-0) Beam

\begin{tabular}{lcccccccc}
\hline \hline $\begin{array}{l}I_{\mathrm{CO}(J=1-0)} \\
\left(\mathrm{mK} \mathrm{km} \mathrm{s}^{-1}\right)\end{array}$ & $\begin{array}{c}I_{\mathrm{CO}(J=2-1)} \\
\left(\mathrm{mK} \mathrm{km} \mathrm{s}^{-1}\right)\end{array}$ & $\begin{array}{c}L_{\mathrm{CO}(J=1-0)} \\
\left(\mathrm{K} \mathrm{km} \mathrm{s}^{-1} \mathrm{pc}^{2}\right)\end{array}$ & $\begin{array}{c}M_{\text {dust }} \\
M_{\odot}\end{array}$ & $\begin{array}{c}M_{\mathrm{H} 2+\mathrm{HI}}^{\text {dust }} \\
10^{7} M_{\odot}\end{array}$ & $\begin{array}{c}M_{\mathrm{H} 2}^{\text {dust }} \\
10^{7} M_{\odot}\end{array}$ & $\begin{array}{c}\alpha_{\mathrm{CO}(J=1-0)} \\
M_{\odot} \mathrm{pc}^{-2}\left(\mathrm{~K} \mathrm{~km} \mathrm{~s}^{-1}\right)^{-1}\end{array}$ & $\begin{array}{c}\mathrm{SFR} \\
10^{-4} M_{\odot} \mathrm{yr}^{-1}\end{array}$ & $\begin{array}{c}L_{8-1000 \mu \mathrm{m}} \\
10^{5} L_{\odot}\end{array}$ \\
\hline $145 \pm 39$ & $<460$ & $3670 \pm 990$ & $771 \pm 184$ & $1.1 \pm 0.3$ & $\begin{array}{c}M_{\mathrm{star}} \\
10^{5} M_{\odot}\end{array}$ \\
\hline
\end{tabular}

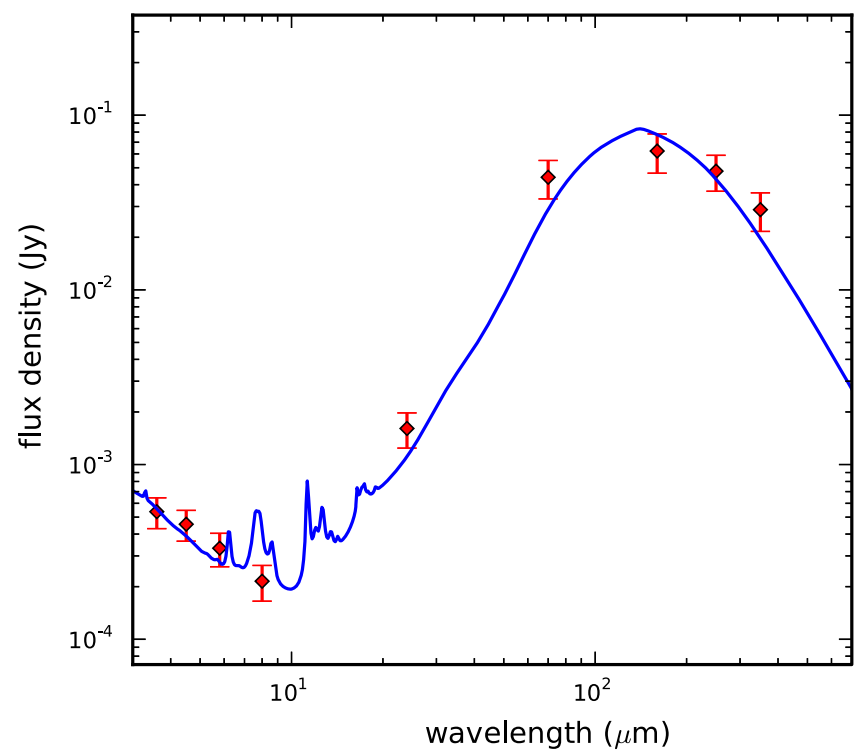

Figure 3. Infrared SED of Sextans A within the IRAM beam is fitted to derive the dust mass. Red symbols are the Spitzer and Herschel photometric points with $1 \sigma$ error bars. The blue solid line indicates the best fit by the dust model (Draine \& Li 2007).

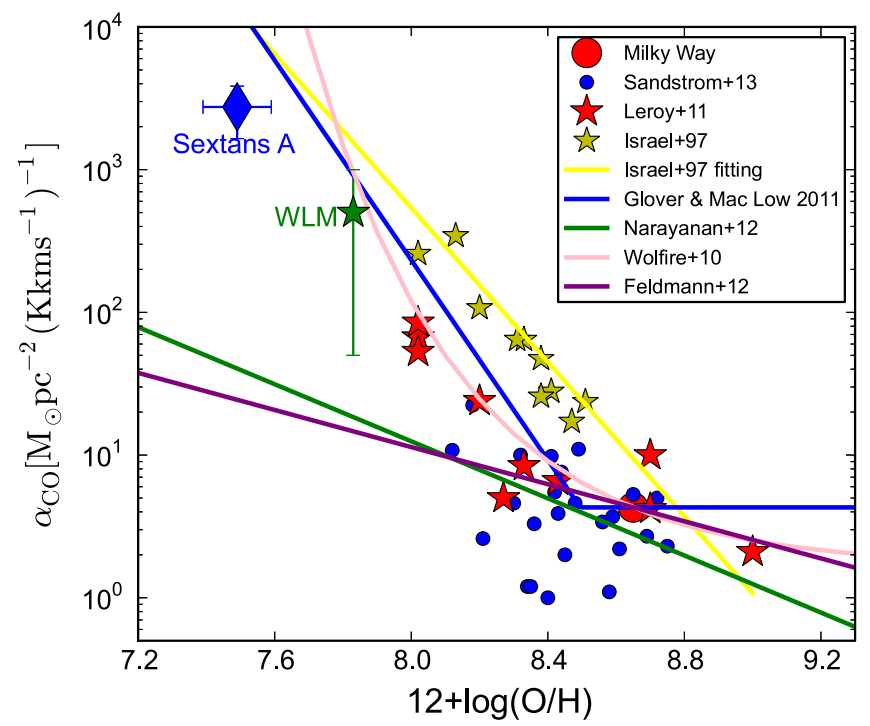

Figure 4. Conversion factor $\left(\alpha_{\mathrm{CO}}\right)$ from the $\mathrm{CO}$ luminosity to the molecular gas mass for Sextans A compared to those at higher metallicities. Symbols are observations in the literature (Israel 1997; Leroy et al. 2011; Elmegreen et al. 2013; Sandstrom et al. 2013) where molecular gas masses are all based on the spatially resolved dust maps except for WLM (see the text for details). Lines are models, either empirical (Israel 1997), or theoretical (Wolfire et al. 2010; Glover \& Mac Low 2011; Feldmann et al. 2012; Narayanan et al. 2012).

quoted here. Our $\alpha_{\mathrm{CO}}$ is much larger than those at higher metallicities. The overall trend of $\alpha_{\mathrm{CO}}$ with metallicity is very steep, with a power-law index between 2.5 and 3 . This implies that $\mathrm{CO}$ emission is very weak in extremely metal-poor galaxies. While our work offers very interesting constraints on $\alpha_{\mathrm{CO}}$ at extremely low metallicity, the result is solely based on one region of $\sim 20$ arcsec compared to the entire galaxy of 5 arcmin. Only about $10 \%$ of the total molecular gas of Sextans A (Shi et al. 2014) is within the beam. Large scatters of $\alpha_{\mathrm{CO}}$ may be expected with future detections of $\mathrm{CO}$ emission over different regions of the galaxy.

The relative strength of the $\mathrm{CO}$ brightness can also be compared to other quantities of Sextans A measured within the IRAM beam as listed in Table 1. By integrating the IR SED of the IRAM region, the $8-1000 \mu \mathrm{m}$ infrared luminosity of dust within the beam is measured to be $1.7 \times 10^{5} L_{\odot}$. So the CO luminosity in $\mathrm{K} \mathrm{km} \mathrm{s}^{-1} \mathrm{pc}^{2}$ relative to the infrared luminosity in $L_{\odot}$ is about $1: 45$, which is slightly larger than spiral galaxies (Genzel et al. 2010). This is partly caused by the rarity of both dust and $\mathrm{CO}$ in extremely metal-poor galaxies, while different excitation mechanisms for $\mathrm{CO}$ and dust must also play some roles. If comparing to the star formation rate (SFR) as measured from the far-UV luminosity (Kennicutt 1998b), the SFR per CO luminosity is about 10 times higher than starforming non-merger massive galaxies (Genzel et al. 2010). If scaling by the stellar mass as measured from Spitzer 3.6 and $4.5 \mu \mathrm{m}$ measurements (Eskew et al. 2012), the CO luminosity per stellar mass of Sextans A within the IRAM beam is on average 10 times lower than non-merger massive galaxies (Genzel et al. 2010).

\section{DISCUSSION}

The detection of CO $J=1-0$ emission in Sextans A, if it is real, offers crucial evidence for the existence of molecular gas in such a low-metallicity environment, confirming the result inferred from the dust measurement (Shi et al. 2014). Although warm $\mathrm{H}_{2}$ has been revealed by infrared spectroscopic observations in several extremely metal-poor galaxies (Hunt et al. 2010), $\mathrm{CO}$ emission, unlike warm $\mathrm{H}_{2}$ that traces shocked regions, is known as a tracer of the ISM where stars form in massive metal-rich galaxies. The detection of CO in Sextans A is a first step toward determining whether $\mathrm{CO}$ can serve a similar role in extremely metal-poor galaxies.

$\mathrm{CO}$ is one of a few efficient coolants of molecular gas in galaxies. In extremely metal-poor galaxies, due to photodissociation, the $\mathrm{CO}$ can only survive in a tiny core of molecular gas clouds, while $\mathrm{H}_{2}$ can be self-shielded and exist in thick envelopes surrounding the $\mathrm{CO}$ molecular core. Thus, cooling through $\mathrm{CO}$ in extremely metal-poor galaxies may be not as effective as in metal-rich galaxies. If the $\mathrm{CO}$ molecular cores in metal-poor galaxies follow the same relationship between the viral masses and $\mathrm{CO}$ luminosities of giant molecular clouds in Milky Way and nearby galaxies (Solomon et al. 1987; Bolatto et al. 2013), the viral mass of the CO molecule core for the observed CO luminosity is estimated to be about $3 \times 10^{4} M_{\odot}$. This is only a tiny fraction of the dustbased total molecular gas mass, as small as $0.3 \%$. Then, the measured $\mathrm{CO}$ within the IRAM beam is associated with only a 
small fraction of the total molecular gas within the same beam, and it cannot effectively cool the bulk of the molecular gas in Sextans A. As a result of the photo-dissociation of CO, atomic or ionized carbon may become abundant. Studies did show that [C II] $158 \mu \mathrm{m}$ is much brighter relative to $\mathrm{CO}$ in dwarfs as compared to in spirals (Brauher et al. 2008; Israel \& Maloney 2011; Madden et al. 2013). With spatially resolved studies of IC 10, Madden et al. (1997) showed that the $\mathrm{H}_{2}$ column density associated with ionized carbon can be five times the observed HI density in order to interpret the cooling as implied by the [C II] luminosity, and the associated $\mathrm{H}_{2}$ mass may be 100 times the mass of $\mathrm{H}_{2}$ associated with $\mathrm{CO}$. However, unlike $\mathrm{CO},\left[\mathrm{C}_{\mathrm{II}}\right]$ with its high excitation temperature $(97 \mathrm{~K})$ still could not cool the gas to the low temperature at which gas may contract to a high density for stars to form.

Figure 4 further shows the comparison between the measured extremely large $\alpha_{\mathrm{CO}}$ of Sextans A with the models' predictions. The empirical relationship (yellow solid line; Israel 1997 ) based on galaxies above $20 \% Z_{\odot}$ predicts that $\alpha_{\mathrm{CO}} \propto$ $Z^{2.7}$, giving a value several times larger than our observed one at $7 \% Z_{\odot}$. Based on the principle that the $\mathrm{CO}$ abundance is primarily regulated by photo-dissociation and the $\mathrm{H}_{2}$ is selfshielded, theoretical models of Glover \& Mac Low (2011) and Wolfire et al. (2010) predict that $\alpha_{\mathrm{CO}}$ is a strong function of the visual extinction for individual clouds. If assuming the visual extinction is proportional to the metallicity, $\alpha_{\mathrm{CO}}$ then increases rapidly with the decreasing metallicity in both models, predicting values larger than those observed for Sextans A as shown in Figure 4. The model of Narayanan et al. (2012) also employed photo-dissociation region models, but for the integrated galaxies instead of individual clouds, and found on average a flat trend of $\alpha_{\mathrm{CO}}$ as a function of metallicity, producing a value much smaller than the observed one at $7 \% Z_{\odot}$. Unlike the above models, the model of Feldmann et al. (2012) employs small-scale magneto-hydrodynamic simulations combined with large-scale simulations of gas distributions to predict $\alpha_{\mathrm{CO}}$ as a function of metallicities. For physical scales of our IRAM beam size (subkiloparsec), a shallow trend as indicated by the purple line in Figure 4 is predicted, with predicted values significantly smaller than the observation.

In summary, current models predict a large range of $\alpha_{\mathrm{CO}}$ at the metallicity of Sextans A. Some are significantly larger than the observed one, while others are much smaller than the observation. It is still difficult to judge which model is better or worse just based on one data point, which is because many assumptions are invoked in theoretical models in order to produce the trend of $\alpha_{\mathrm{CO}}$ as a function of metallicity. A large range of the model's predictions reflects the limited knowledge about $\mathrm{CO}$ formation and destruction, as well as properties of gas clouds over different spatial scales at the extreme low metallicity. If $\alpha_{\mathrm{CO}}$ is truly large as seen in this study, CO may not be an efficient coolant in metal-poor galaxies, and its application as a tracer of the molecular gas mass may be inappropriate in the early universe.

\section{CONCLUSIONS}

We reported a marginal detection of $\mathrm{CO} J=1-0$ emission by targeting the brightest far-IR peak in an extremely metalpoor galaxy, Sextans A. The signal is about $10 \mathrm{~km} \mathrm{~s}^{-1}$ offset from the HI peak within the same beam. The $\alpha_{\mathrm{CO}}$ is further derived by comparing the estimated $\mathrm{CO}$ flux to the $\mathrm{H}_{2}$ mass, as inferred from the dust map. In spite of a marginal signal, the observation is deep enough to play a strong limit on the $\alpha_{\mathrm{CO}}$ that is about 700 times the Milky Way value. This suggests that $\mathrm{CO}$ emission is exceedingly weak in extremely metal-poor galaxies, challenging its role as a gas coolant in these galaxies. Current theoretical models produce a large range of $\alpha_{\mathrm{CO}}$ at the metallicity of Sextans A.

We thank the anonymous referee for constructive comments that significantly improved the paper. Y.S. acknowledges support for this work from Natural Science Foundation of China under grant 11373021 and by the Strategic Priority Research Program "The Emergence of Cosmological Structures" of the Chinese Academy of Sciences, grant No. XDB09000000. Y.G. is supported under grants 11390373, 11420101002, and XDB09000000. Z.Y.Z. acknowledges support from the European Research Council (ERC) in the form of an Advanced Grant, COSMICISM.

\section{REFERENCES}

Aniano, G., Draine, B. T., Gordon, K. D., \& Sandstrom, K. 2011, PASP, 123,1218

Bolatto, A. D., Wolfire, M., \& Leroy, A. K. 2013, ARA\&A, 51, 207

Brauher, J. R., Dale, D. A., \& Helou, G. 2008, ApJS, 178, 280

Dale, D. A., Cohen, S. A., Johnson, L. C., et al. 2009, ApJ, 703, 517

Draine, B. T., \& Li, A. 2007, ApJ, 657, 810

Elmegreen, B. G., Rubio, M., Hunter, D. A., et al. 2013, Natur, 495, 487

Eskew, M., Zaritsky, D., \& Meidt, S. 2012, AJ, 143, 139

Feldmann, R., Gnedin, N. Y., \& Kravtsov, A. V. 2012, ApJ, 747, 124

Gao, Y., \& Solomon, P. M. 2004, ApJ, 606, 271

Genzel, R., Tacconi, L. J., Gracia-Carpio, J., et al. 2010, MNRAS, 407, 2091

Glover, S. C. O., \& Mac Low, M.-M. 2011, MNRAS, 412, 337

Goldsmith, P. F. 2001, ApJ, 557, 736

Hunt, L. K., Thuan, T. X., Izotov, Y. I., \& Sauvage, M. 2010, ApJ, 712, 164 Israel, F. P. 1997, A\&A, 328, 471

Israel, F. P., \& Maloney, P. R. 2011, A\&A, 531, AA19

Kennicutt, R. C., Jr. 1998a, ApJ, 498, 541

Kennicutt, R. C., Jr. 1998b, ARA\&A, 36, 189

Kniazev, A. Y., Grebel, E. K., Pustilnik, S. A., Pramskij, A. G., \& Zucker, D. B. 2005, AJ, 130, 1558

Leroy, A. K., Bolatto, A., Gordon, K., et al. 2011, ApJ, 737, 12

Leroy, A., Cannon, J., Walter, F., Bolatto, A., \& Weiss, A. 2007, ApJ, 663, 990

Madden, S. C., Poglitsch, A., Geis, N., Stacey, G. J., \& Townes, C. H. 1997, ApJ, 483, 200

Madden, S. C., Rémy-Ruyer, A., Galametz, M., et al. 2013, PASP, 125, 600

Narayanan, D., Krumholz, M. R., Ostriker, E. C., \& Hernquist, L. 2012, MNRAS, 421, 3127

Ott, J., Stilp, A. M., Warren, S. R., et al. 2012, AJ, 144, 123

Pettini, M., \& Pagel, B. E. J. 2004, MNRAS, 348, L59

Rémy-Ruyer, A., Madden, S. C., Galliano, F., et al. 2014, A\&A, 563, A31

Sandstrom, K. M., Leroy, A. K., Walter, F., et al. 2013, ApJ, 777, 5

Shi, Y., Armus, L., Helou, G., et al. 2014, Natur, 514, 335

Solomon, P. M., Rivolo, A. R., Barrett, J., \& Yahil, A. 1987, ApJ, 319, 730

Taylor, C. L., Kobulnicky, H. A., \& Skillman, E. D. 1998, AJ, 116, 2746

Wolfire, M. G., Hollenbach, D., \& McKee, C. F. 2010, ApJ, 716, 1191

Young, J. S., \& Scoville, N. 1982, ApJ, 258, 467 